\title{
Adverse events associated with treatment of latent tuberculosis in the general population
}

\author{
Benjamin M. Smith MD, Kevin Schwartzman MD MPH, Gillian Bartlett PhD, Dick Menzies MD MSc
}

\begin{abstract}
Background: Guidelines recommend treatment of latent tuberculosis in patients at increased risk for active tuberculosis. Studies investigating the association of therapy with serious adverse events have not included the entire treated population nor accounted for comorbidities or occurrence of similar events in the untreated general population. Our objective was to estimate the risk of adverse events requiring hospital admission that were associated with therapy for latent tuberculosis infection in the general population.
\end{abstract}

Methods: Using administrative health data from the province of Quebec, we created a historical cohort of all residents dispensed therapy for latent tuberculosis between 1998 and 2003. Each patient was matched on age, sex and postal region with two untreated residents. The observation period was 18 months (from 6 months before to 12 months after initiation of therapy). The primary outcome was hospital admission for therapy-associated adverse events.

Results: During the period of observation, therapy for latent tuberculosis was dispensed to 9145 residents, of whom $95 \%$ started isoni- azid and 5\% started rifampin. Pretreatment comorbid illness was significantly more common among patients receiving such therapy compared with the matched untreated cohort. Of all patients dispensed therapy, 45 $(0.5 \%)$ were admitted to hospital for a hepatic event compared with $15(0.1 \%)$ of the untreated patients. For people over age 65 years, the odds of hospital admission for a hepatic event among patients treated for latent tuberculosis infection was significantly greater than among matched untreated people after adjustment for comorbidities (odds ratio [OR] 6.4, 95\% Cl 2.2-18.3). Excluding patients with comorbid illness, there were two excess admissions to hospital for hepatic events per 100 patients initiating therapy compared with the rate among untreated people over 65 years $(95 \% \mathrm{Cl} 0.1-3.87)$.

Interpretation: The risk of adverse events requiring hospital admission increased significantly among patients over 65 years receiving treatment for latent tuberculosis infection. The decision to treat latent tuberculosis infection in elderly patients should be made after careful consideration of risks and benefits.
$\mathrm{T}$ reatment of latent tuberculosis infection can effectively reduce the risk of development of active tuberculosis ${ }^{1,2}$ and is considered an essential component of tuberculosis control in low-incidence countries such as the United States and Canada. ${ }^{3}$ The decision to treat a patient who has latent tuberculosis infection depends primarily on the balance between the likelihood of development of active disease and the risk of therapy-related adverse events. ${ }^{1,2}$

In 2007, 50\% of active cases of tuberculosis in the United States occurred in patients over 45 years of age. ${ }^{4}$ In part, this rate reflects the higher prevalence of many risk factors for reactivation of latent tuberculosis infection with older age (e.g., end-stage renal disease, diabetes mellitus, cancer and chemotherapy). Current recommendations emphasize treatment of people with latent tuberculosis infection who have one or more of these risk factors, regardless of age. ${ }^{1,2}$ However, such treatment for older patients remains controversial, and physician practice reflects concern about age-related adverse events. In one study, age was the most commonly cited reason that therapy for latent tuberculosis infection was not started for patients over 50 years. ${ }^{5}$

Although several randomized trials showed an excellent safety profile for isoniazid, ${ }^{6}$ subsequent surveillance studies reported an overall incidence of probable isoniazid-related clinical hepatitis of $0.7 \%$, which was associated with older age and with mortality of $0.1 \%$ among those over age 35 years. ${ }^{78}$ However, it was later noted that seven of the eight deaths with isoniazid therapy occurred in one city, which had experienced a coincident increase in viral hepatitis. ${ }^{9}$ These factors highlight the importance of an untreated reference popula-
Competing interests: None declared.

This article has been peer reviewed.

Correspondence to: Dr. Dick Menzies; dick.menzies@mcgill.ca

CMAJ 2011. DOI:10.1503 /cmaj.091824 
tion. A subsequent controlled trial showed an agerelated increase in hepatic events independent of comorbidity, but ascertainment and severity of these events was unclear. ${ }^{10}$ More recently, large observational studies from specialized tuberculosis clinics have reported low rates of adverse events, although rates remain higher among older people. ${ }^{11-14}$ However, rates of comorbidities were not reported ${ }^{11,13}$ or were incomplete..$^{12,14}$ Ascertainment of adverse events may have been incomplete as well, given that patients treated by private health care providers were not included in these surveys. ${ }^{15}$

The primary objective of our study was to derive population-based estimates of rates of severe adverse events associated with therapy for latent tuberculosis infection in different age groups.

\section{Methods}

Quebec is a Canadian province with a population of 7.7 million people. ${ }^{16}$ The health insurance board of Quebec (the Régie de l'Assurance Maladie du Québec, or RAMQ) is the governmentmanaged health care insurer for over $99 \%$ of permanent residents. Importantly, since January 1997, the RAMQ has also provided all antituberculosis medications free of charge to Quebec residents, regardless of private insurance.

\section{Data sources}

We extracted depersonalized data from provincial health administrative databases, including demographic characteristics, health-related services accessed, diagnostic codes and pharmacydispensed therapy for tuberculosis. Data on acute-care admissions to hospital were obtained from the Med-Echo database, which includes dates of admission and discharge and up to eight discharge diagnoses. Information on acute-care hospital admissions (including discharge diagnoses) is extracted by trained archivists and coded using the International Statistical Classification of Injuries and Causes of Death, 9th revision (ICD-9). The databases are the basis for provider reimbursement, and the accuracy of discharge diagnoses have been validated for epidemiologic applications. ${ }^{17,18}$

A cohort was constructed of all patients registered as RAMQ beneficiaries who were dispensed at least 30 days of therapy for latent tuberculosis infection between Jan. 1, 1998, and Dec. 31, 2003. For each patient, the period of observation was six months before to 12 months after the date of treatment initiation, which was considered the index date. Regimens that were considered therapy for latent tuberculosis infection were isoniazid alone, rifampin alone or, in the event of regimen change, sequential use of isoniazid and rifampin. ${ }^{1,2}$ These remain the only guideline-recommended regimens for latent tuberculosis infection. ${ }^{1,2}$ Patients dispensed rifampin with an alternate indication (e.g., leprosy or chronic bacterial infection such as osteomyelitis or infected prosthesis) were excluded. Those dispensed rifampin and pyrazinamide simultaneously were also excluded, because this regimen is no longer recommended. ${ }^{1,2,819}$ Prescription for at least 30 days of treatment was required for inclusion because rifampin may be used for other indications (e.g., prophylaxis of meningitis) and such uses are usually for less than one month. The period during which patients were considered susceptible to outcomes of interest started on the date of the first dispensation of therapy for latent tuberculosis infection and ended 60 days after the last pharmacy visit.

For each person dispensed a treatment regimen for latent tuberculosis infection (the latent tuberculosis infection [LTBI] cohort), two RAMQ beneficiaries were identified who had not received tuberculosis drugs during the corresponding period (the untreated cohort). They were matched by age (within five years), sex and postal region, with a median population in each postal region of $16336 .{ }^{16}$ The time at risk for each untreated patient was matched to the corresponding treatment period for LTBI patients using the index date.

Demographic characteristics of patients were abstracted from the RAMQ database. Using the previously validated Charlson score adapted for administrative databases,$^{20}$ comorbid illnesses were identified from ICD-9 codes for all hospital and outpatient visits during the six months before therapy (or calendar-matched period for untreated patients).

\section{Outcomes}

The primary outcome was serious adverse events, defined as acute-care hospital admissions for which the discharge diagnoses included at least one of five predefined conditions considered potentially related to therapy for latent tuberculosis infection. These five conditions were hepatic (e.g., noninfectious or toxic hepatitis), gastrointestinal (e.g., dyspepsia, vomiting), hematologic (e.g., thrombocytopenia), allergy (e.g., dermatitis) or poisoning. For specific ICD-9 codes, see Appendix 1 (available at www.cmaj.ca/cgi/content/full /cmaj.091824/DC1). For comparisons made within the LTBI cohort, serious adverse events were considered probably related to therapy if hospital admission occurred within 60 days of pharmacy dispensation of isoniazid or rifampin, and if that drug was discontinued permanently thereafter. A period of 60 days after the pharmacy visit was per- 
mitted because of the routine dispensation of 30 days worth of medication and the allowance for missed doses resulting in extended exposure to the therapy. Because each of the five conditions can have multiple causes besides therapy for latent tuberculosis infection, if a person was admitted to hospital for the identical ICD-9 code during the six months before therapy was started, it was not considered a therapy-related event.

To determine the background rate of hospital admission for ICD-9 codes of interest, severe clinical events were also defined for the untreated cohort. Every untreated patient was assigned the calendar period of susceptibility corresponding to their matched treated patient. Similar to the treatment cohort, hospital admission for any of the five conditions during this period was considered a serious clinical event if it did not occur during the six months before susceptibility.

Therapy was considered completed if the total doses dispensed corresponded to six months of isoniazid or four months of rifampin. ${ }^{1,2}$ Change in therapy was defined as the sequential, nonoverlapping dispensing of isoniazid or rifampin.

\section{Statistical analysis}

Conditional logistic regression was used for comparisons between patients with latent tuberculosis infection and matched untreated cohorts, and was expressed using 95\% confidence intervals (CIs). Potential confounding variables included in the analysis were previous hospital admission, Charlson comorbidity score and the following individual components of the Charlson score: cancer, diabetes, HIV infection, and liver, renal and vascular disease. The Mantel-Haenszel method was used to calculate age-stratified risk differences between treated and untreated cohorts - for all patients and the subset without comorbidity. For comparisons within the LTBI cohort, multivariate logistic regression was used.

This study was approved by the research ethics board of the McGill University Health Centre.

\section{Results}

Between January 1, 1998, and December 31, 2003, 9145 people were dispensed at least 30 days worth of isoniazid or rifampin alone or

Table 1: Characteristics of cohort receiving therapy for latent tuberculosis infection and untreated cohort

\begin{tabular}{|c|c|c|c|c|}
\hline \multirow[b]{2}{*}{ Characteristic } & \multicolumn{3}{|c|}{ Cohort receiving LTBI therapy, no. (\%) } & \multirow{2}{*}{$\begin{array}{c}\begin{array}{c}\text { Untreated cohort, * } \\
\text { no. }(\%)\end{array} \\
\text { Total } \\
n=18290\end{array}$} \\
\hline & $\begin{array}{c}\text { Total } \\
n=9145\end{array}$ & $\begin{array}{l}\text { Isoniazidt } \\
n=8686\end{array}$ & $\begin{array}{c}\text { Rifampint } \\
n=459\end{array}$ & \\
\hline \multicolumn{5}{|l|}{ Age, yr } \\
\hline$\leq 35$ & $4523(49.5)$ & $4356(50.1)$ & $167(36.4)$ & $9046 \quad(49.5)$ \\
\hline $36-50$ & $2533(27.7)$ & 2408 (27.7) & $125(27.2)$ & $5066 \quad(27.7)$ \\
\hline $51-65$ & $1232(13.5)$ & 1159 (13.3) & 73 (15.9) & 2464 (13.5) \\
\hline$>65$ & $857 \quad(9.4)$ & $763 \quad(8.9)$ & $94(20.5)$ & $1714 \quad(9.4)$ \\
\hline Sex, female & $5000(54.7)$ & $4784(55.1)$ & $216(47.1)$ & $10000 \quad(54.7)$ \\
\hline Residence, urban $\ddagger$ & $6216(68.0)$ & $5913(68.1)$ & $295(64.3)$ & $12432(68.0)$ \\
\hline $\begin{array}{l}\geq 1 \text { hospital admissions } \\
\text { in previous } 6 \mathrm{mo} \S\end{array}$ & $946(10.3)$ & $866(10.0)$ & $80(17.4)$ & $(4.0)$ \\
\hline \multicolumn{5}{|l|}{ Comorbid illness§ণ } \\
\hline Liver disease & $54 \quad(0.6)$ & $45 \quad(0.5)$ & $9 \quad(2.0)$ & $17 \quad(0.1)$ \\
\hline Kidney disease & $171 \quad(1.9)$ & $156 \quad(1.8)$ & $15 \quad(3.3)$ & $(0.1)$ \\
\hline Diabetes & $229 \quad(2.5)$ & $211 \quad(2.4)$ & $18 \quad(3.9)$ & (1.5) \\
\hline HIV infection or AIDS & $50 \quad(0.6)$ & $48 \quad(0.6)$ & $2 \quad(0.4)$ & $(0.0)$ \\
\hline Malignancy & $287 \quad(3.1)$ & $267 \quad(3.1)$ & $20 \quad(4.4)$ & $(1.2)$ \\
\hline Peptic ulcer disease & $53 \quad(0.6)$ & $46 \quad(0.5)$ & $7 \quad(1.5)$ & $39 \quad(0.2)$ \\
\hline $\begin{array}{l}\text { Chronic pulmonary } \\
\text { disease }\end{array}$ & $786 \quad(8.6)$ & $744 \quad(8.7)$ & $42 \quad(9.2)$ & (3.9) \\
\hline \multicolumn{5}{|c|}{$\begin{array}{l}\text { Note: LTBI = latent tuberculosis infection. } \\
\text { *Patients in the untreated cohort were matched by age, sex and postal region in a 2:1 ratio to those in the LTBI cohort. } \\
\text { tIf a change in regimen occurred during the course of therapy, patients were classified according to the initial regimen } \\
\text { dispensed. } \\
\text { fUrban residency was defined according to postal-code region. } \\
\text { \$Hospital admission for any reason and comorbid illness were assessed during the six months before starting LTBI therapy (or a } \\
\text { matched calendar period for control patients). } \\
\text { qAll differences in prevalence of comorbid illness between the total treatment cohort and the control cohort were significant at } \\
p<0.01 \text {. }\end{array}$} \\
\hline
\end{tabular}


sequentially. For each of these patients, two additional RAMQ beneficiaries were identified who were of the same age group, sex and postal region $(n=18290)$ and who did not receive any form of tuberculosis therapy during the same period.

\section{Rates of hospital admission among treated patients and matched controls}

As shown in Table 1, slightly more than half of all patients dispensed therapy for latent tuberculosis infection were over 35 years old $(n=4622)$. Matching of LTBI patients and control patients resulted in an identical proportion of women $(54.7 \%)$, urban residents $(68 \%)$, median house- hold income by postal region ( $\$ 43000)$ and percentage of college and university graduates by postal region (33\%). Among patients dispensed latent tuberculosis infection therapy, isoniazid was the most common intial regimen (92.8\%), whereas rifampin usage increased with patient age. Rates of completion were similar for six months of isoniazid (54.1\%) and four months of rifampin $(56.2 \%)$. Patients in the LTBI cohort had a significantly higher prevalence of comorbid diseases than untreated patients (Table 1). Rates of hospital admission during the six months before starting therapy were also higher among patients with latent tuberculosis infection compared with those in the untreated cohort.

Table 2: Event rates and odds ratios for outcomes of interest, by cohort

Crude event rate, events/total (rate per 100 patients)
Event ratio, cohort treated for LTBI v. untreated cohort $(95 \% \mathrm{Cl})$

\begin{tabular}{|c|c|c|c|c|c|}
\hline Outcome; age, yr & LTBI therapy cohort & Untreated cohort* & Crude ORT & Adjusted OR & Adjusted OR§ \\
\hline \multicolumn{6}{|c|}{ Hospital admission for any reasonๆ } \\
\hline Total & 1046/9145 (11.4) & $1474 / 18290$ & $1.4(1.3-1.5)$ & $1.1 \quad(1.0-1.2)$ & $1.1(1.0-1.2)$ \\
\hline$\leq 35$ & $264 / 4523 \quad(5.8)$ & $585 / 9046 \quad(6.5)$ & $0.9(0.8-1.0)$ & $0.8 \quad(0.7-1.0)$ & $0.8(0.7-1.0)$ \\
\hline $36-50$ & $255 / 2533(10.1)$ & $370 / 5066 \quad(7.3)$ & $1.4(1.2-1.6)$ & $1.2(1.0-1.4)$ & $1.2(1.0-1.4)$ \\
\hline $51-65$ & $201 / 1232(16.3)$ & $202 / 2464$ (8.2) & $2.0(1.6-2.4)$ & $1.3(1.0-1.6)$ & $1.3(1.1-1.7)$ \\
\hline$>65$ & $326 / 857(38.0)$ & $317 / 1724(18.4)$ & $2.1(1.8-2.4)$ & $1.6(1.3-1.9)$ & $1.5(1.3-1.9)$ \\
\hline
\end{tabular}

Hospital admission for any outcome of

interest (hepatic, allergic, Gl, hematologic or poisoning)§

\begin{tabular}{lrlrlllll} 
Total & $121 / 9145$ & $(1.3)$ & $50 / 18290$ & $(0.3)$ & $4.8(3.5-6.7)$ & 2.8 & $(2.0-4.2)$ & $2.9(2.0-4.3)$ \\
$\leq 35$ & $17 / 4523$ & $(0.4)$ & $9 / 9046$ & $(0.1)$ & $3.8(1.7-8.5)$ & 4.4 & $(1.5-12.7)$ & $4.4(1.5-12.7)$ \\
\hline $36-50$ & $27 / 2533$ & $(1.1)$ & $17 / 5066$ & $(0.3)$ & $3.2(1.7-5.8)$ & 2.5 & $(1.2-5.2)$ & $2.5(1.2-5.3)$ \\
$51-65$ & $26 / 1232$ & $(2.1)$ & $10 / 2464$ & $(0.4)$ & $5.2(2.5-10.8)$ & 2.6 & $(1.0-6.3)$ & $2.2(0.8-6.4)$ \\
$>65$ & $51 / 857$ & $(6.0)$ & $14 / 1714$ & $(0.8)$ & $7.3(4.0-13.2)$ & 4.0 & $(2.0-7.9)$ & $3.8(1.8-7.8)$
\end{tabular}

Hospital admission for hepatic event of interest§

\begin{tabular}{|c|c|c|c|c|c|c|}
\hline Total & $45 / 9145$ & $(0.5)$ & $15 / 18290 \quad(0.1)$ & $6.5(3.8-11.1)$ & $3.7 \quad(2.0-6.9)$ & $2.7(1.3-5.6)$ \\
\hline$\leq 35$ & $5 / 4523$ & $(0.1)$ & $1 / 9046 \quad(0.0)$ & $10.0(1.2-85.6)$ & NC & NC \\
\hline $36-50$ & $8 / 2533$ & $(0.3)$ & $7 / 5066 \quad(0.1)$ & $2.6(1.0-6.9)$ & $2.0(0.6-6.9)$ & $1.5(0.4-5.6)$ \\
\hline $51-65$ & $10 / 1232$ & $(0.8)$ & $4 / 2464 \quad(0.2)$ & $7.0(2.3-21.3)$ & $2.9(0.7-13.0)$ & $2.6(0.4-16.0)$ \\
\hline$>65$ & $22 / 857$ & (2.6) & $3 / 1714 \quad(0.2)$ & $10.8(4.2-28.0)$ & $6.4(2.2-18.3)$ & $3.2(0.9-11.7)$ \\
\hline Change in regimen** & $226 / 9145$ & (2.5) & NA & NA & NA & NA \\
\hline Death from any cause§ & $105 / 9145$ & (1.1) & $72 / 18290 \quad(0.4)$ & $2.9(2.2-3.9)$ & $1.3(0.9-2.0)$ & $1.4(1.0-2.1)$ \\
\hline $\begin{array}{l}\text { Death within } 30 \text { days after } \\
\text { any outcome of interest }\end{array}$ & $7 / 9145$ & $(0.1)$ & $3 / 18290 \quad(0.0)$ & $5.3(1.4-20.1)$ & $4.8(0.8-27.7)$ & $1.4(0.1-23.6)$ \\
\hline
\end{tabular}

Note: $\mathrm{Cl}=$ confidence interval; $\mathrm{GI}=$ gastrointestinal; $\mathrm{LTBI}=$ latent tuberculosis infection; $\mathrm{NA}=$ not applicable; $\mathrm{NC}=$ no convergence; OR = odds ratio.

*An outcome of interest in the untreated cohort refers to an event meeting the same definition as that for LTBI cohort that occurred after the index date for the matched untreated cohort.

tORs were calculated using conditional logistic regression. Cohorts were matched by age and sex.

fLogistic model variables were Charlson score, previous hospital admission (one or more hospital admissions in the six months before starting LTBI therapy or matched period for the untreated cohort).

§Logistic model variables were previous hospital admission (one or more hospital admissions in the six months before starting LTBI therapy or matched period for the untreated cohort), liver disease, diabetes, renal insufficiency, malignancy, vascular disease and HIV infection or AIDS.

ПOutcomes were assessed from the first dispensed dose of LTBI therapy to 60 days after the last dispensed prescription (or matched calendar period for controls). ** Sequential, nonoverlapping use of isoniazid and rifampin (198/8686 isoniazid to rifampin; 28/459 rifampin to isoniazid). 
In total, 121 patients who received therapy for latent tuberculosis infection and 50 untreated patients were admitted to hospital for events of interest, including 45 patients from the LTBI cohort and 15 untreated patients who were admitted for a hepatic event (Table 2). The risk of experiencing a hepatic event was greatest among people over age 65 years who were treated for latent tuberculosis infection (2.6 per 100 patients dispensed therapy). After adjustment for previous hospital admission and Charlson comorbidity score, patients receiving treatment for latent tuberculosis infection who were over age 65 years had significantly increased odds for a hepatic event (adjusted odds ratio [OR] 6.4, 95\% CI 2.2-18.3). As shown in Table 3, the crude risk of events of interest among people without comorbidity over age 65 years was significantly higher among those treated for latent tuberculosis infection than those untreated $(2.7$ excess events per 100 persons treated); most of this excess risk was accounted for by hepatic events (Table 4). Median duration of hospital stay for an adverse event was seven days (interquartile range [IQR] 3-20 days) for patients with latent tuberculosis infection compared with five days (IQR 2-10 days) for untreated patients.

Seven patients who received therapy for latent tuberculosis infection and three control patients died within one month of an event of interest. However, after detailed analysis, it was difficult to attribute these deaths directly to therapy for latent tuberculosis infection. Three patients who were dispensed therapy underwent liver transplant within five months of admission to hospital for a hepatic adverse event. Patients were 55-64 years old and had accessed health care for liver disease before being given therapy for latent tuberculosis infection. None of the control cohort members underwent liver transplant.

Table 3: Crude risk estimates for any event of interest requiring admission to hospital, by cohort

\begin{tabular}{|c|c|c|c|c|c|c|}
\hline Age group, yr & \multicolumn{2}{|c|}{$\begin{array}{l}\text { LTBI therapy cohort, no. of } \\
\text { events/total (rate per } 100 \text { patients) }\end{array}$} & \multicolumn{2}{|c|}{$\begin{array}{l}\text { Untreated cohort, no. of } \\
\text { events/total (rate per } 100 \text { patients)* }\end{array}$} & \multicolumn{2}{|c|}{$\begin{array}{l}\text { Risk difference, cohort treated } \\
\text { for LTBI v. untreated cohort, } \\
\text { per } 100 \text { patients treated }(95 \% \mathrm{Cl})\end{array}$} \\
\hline $36-50$ & 27/2533 (1.1) & 10/1898 (0.5) & $17 / 5066(0.3)$ & 10/3796 (0.3) & 0.7 (0.3 to 1.2$)$ & 0.3 (-0.1 to 0.6$)$ \\
\hline $51-65$ & 26/1232 (2.1) & 4/668 (0.6) & $10 / 2464(0.4)$ & 4/1336 (0.3) & 1.7 (0.9 to 2.5$)$ & $0.3(-0.4$ to 1.0$)$ \\
\hline$>65$ & $51 / 857(6.0)$ & $7 / 205$ (3.4) & 14/1714 (0.8) & $3 / 410(0.7)$ & 5.1 (3.5 to 6.8 ) & $2.7 \quad(0.1$ to 5.3$)$ \\
\hline
\end{tabular}

Table 4: Crude risk estimates for hepatic events requiring admission to hospital, by cohort

\begin{tabular}{|c|c|c|c|c|c|c|}
\hline \multirow[b]{2}{*}{ Age group, yr } & \multicolumn{2}{|c|}{$\begin{array}{l}\text { LTBI therapy cohort, } \\
\text { no. of events/total } \\
\text { (rate per } 100 \text { patients) }\end{array}$} & \multicolumn{2}{|c|}{$\begin{array}{l}\text { Untreated cohort, no. of } \\
\text { events/total (rate per } 100 \text { patients)* }\end{array}$} & \multicolumn{2}{|c|}{$\begin{array}{c}\text { Risk difference, cohort treated for LTBI } \\
\text { v. untreated cohort, per } 100 \text { patients } \\
\text { treated }(95 \% \mathrm{Cl})\end{array}$} \\
\hline & $\begin{array}{l}\text { All patients } \\
\text { treated for } \\
\text { LTBI }\end{array}$ & $\begin{array}{l}\text { Patients without } \\
\text { comorbidity } \\
\text { treated for LTBIt }\end{array}$ & $\begin{array}{l}\text { All untreated } \\
\text { patients }\end{array}$ & $\begin{array}{l}\text { Untreated } \\
\text { patients without } \\
\text { comorbidity }\end{array}$ & All patients & $\begin{array}{l}\text { Patients without } \\
\text { comorbidity } \dagger\end{array}$ \\
\hline Total & $45 / 9145(0.5)$ & $15 / 6536(0.2)$ & 15/18 $290(0.1)$ & $6 / 13072(0.0)$ & $0.4 \quad(0.3$ to 0.6$)$ & $0.2 \quad(0.1$ to 0.3$)$ \\
\hline$\leq 35$ & $5 / 4523(0.1)$ & 5/3765 (0.1) & 1/9046 (0.0) & $0 / 7530(0.0)$ & $0.1 \quad$ (0.0 to 0.2$)$ & $0.1 \quad(0.0$ to 0.2$)$ \\
\hline $36-50$ & $8 / 2533(0.3)$ & 4/1898 (0.2) & $7 / 5066(0.1)$ & $5 / 3796(0.1)$ & $0.2(-0.1$ to 0.4$)$ & $0.1(-0.2$ to 0.3$)$ \\
\hline $51-65$ & $10 / 1232(0.8)$ & $2 / 668(0.3)$ & $4 / 2464(0.2)$ & $1 / 1336(0.1)$ & $0.6 \quad(0.1$ to 1.2$)$ & $0.2(-0.2$ to 0.7$)$ \\
\hline$>65$ & $22 / 857(2.6)$ & $4 / 205(2.0)$ & $3 / 1714(0.2)$ & $0 / 410(0.0)$ & 2.4 (1.3 to 3.5$)$ & $2.0 \quad(0.1$ to 3.9$)$ \\
\hline
\end{tabular}




\section{Factors associated with serious adverse} events among treated patients

Within the LTBI cohort, the overall unadjusted rates of serious adverse events requiring hospital admission were 0.8 (isoniazid) and 1.1 (rifampin) per 100 person-years. Rifampin was used more often in older patients with more comorbidities. After adjustment for these potentially confounding factors, the risk of hepatic events was nonsignificantly higher with isoniazid (OR for isoniazid v. rifampin $=2.1,95 \%$ CI 0.3-15.5).

Hospital admission, any physician visits for liver disease or a higher Charlson comorbidity score during the six months before the index date (i.e., start of treatment for latent tuberculosis infection) were significantly associated with subsequent hepatic events in multivariate analysis (Appendix 2, available at www.cmaj.ca/cgi /content/full/cmaj.091824/DC1).

In this study, 763 patients older than age 65 years (including 156 over age 80 years) received isoniazid therapy, allowing age-stratified estimates of probable isoniazid-associated severe hepatic adverse events. As shown in Table 5, relative to those under the age of 35 and with adjustment for comorbidities, the risk of hepatic adverse events increased significantly with age, with an approximate seven-fold increase among patients aged 50 to 65 years and a 35 -fold increase among patients over age 65 years.

\section{Interpretation}

In our population-based observational study, there was an excess risk of serious adverse events requiring hospital admission associated with therapy for latent tuberculosis infection compared with that in the general population. The excess risk was contributed largely by hepatic events among patients over age 65 years and remained significant after adjustment for comorbidity score (adjusted OR 6.4, 95\% CI 2.2-18.3). Among patients in the same age group, exclusion of those with pre-existing comorbid illnesses yielded 2.7 excess events per 100 persons initiating therapy. Current North American guidelines recommend treatment of patients with latent tuberculosis infection who have increased risk of reactivation regardless of age. ${ }^{1,2}$ Our results highlight a substantial excess risk of therapy for latent tuberculosis infection among patients over 65 .

\section{Strengths and limitations}

A major strength of this study, in contrast to previous observational studies, ${ }^{11-15,21-24}$ was its complete population coverage. The study population was diverse with respect to comorbidities, health care providers and age, with more than half of patients over age 35 years. The administrative databases include information on health care services and hospital admissions for more than $99 \%$ of people resident in Quebec; hence outcome ascertainment was relatively complete. Since tuberculosis therapy is provided to all people through the public health care system, every legal resident of the province who received therapy for latent tuberculosis infection should have been included.

Studies using health administrative databases have inherent limitations in the accuracy of identification of treatment, comorbidities and outcomes from diagnostic codes. However, the treatment for latent tuberculosis infection is very specific. The outcomes were defined based on hospital discharge diagnoses, which are abstracted and coded by trained archivists. The accuracy of this approach has been validated previously for this database..$^{18}$ As well, we restricted the analysis to events that occurred after at least 30 doses were dispensed. If anything, this restriction may have led to an underestimation of adverse events, because events that occurred in the fourth month of rifampin therapy or in the sixth or later months of isoniazid therapy would not have met our outcome definition.

Prior observational studies of adverse events with latent tuberculosis infection therapy have

\begin{tabular}{|c|c|c|c|c|}
\hline $\begin{array}{l}\text { Age } \\
\text { group, yr }\end{array}$ & $\begin{array}{l}\text { No. of } \\
\text { events/total } \\
\text { (risk per } 100 \\
\text { patients) }\end{array}$ & $\begin{array}{l}\text { Crude OR } \\
(95 \% \mathrm{Cl})\end{array}$ & $\begin{array}{l}\text { OR adjusted for sex } \\
\text { and prior liver disease } \\
\qquad(95 \% \mathrm{Cl})\end{array}$ & $\begin{array}{c}\text { OR adjusted for sex } \\
\text { and Charlson score } \\
\qquad(95 \% \mathrm{Cl})\end{array}$ \\
\hline$\leq 35$ & 2/4356 (0.05) & 1.00 (reference) & 1.00 (reference) & 1.00 (reference) \\
\hline $36-50$ & $3 / 2408(0.1)$ & $2.7 \quad(0.5-16.3)$ & $2.7 \quad(0.5-16.0)$ & $1.3(0.2-10.0)$ \\
\hline $51-65$ & $5 / 1159(0.4)$ & $9.4 \quad(1.8-48.7)$ & $5.7 \quad(1.0-33.7)$ & $6.7(1.2-39.2)$ \\
\hline$>65$ & 14/763 (1.8) & $40.7 \quad(9.2-179.5)$ & $34.2 \quad(7.6-153.8)$ & $34.5(7.0-170.2)$ \\
\hline
\end{tabular}


failed to systematically account for comorbidities. ${ }^{11-15,21-24}$ In addition, all of these studies were case series, so they could not account for the background occurrence of similar, coincidental health events in the general population. We included two untreated persons matched by age, sex and postal region, allowing us to better account for the rate of similar, coincidental health events that might be expected. We also assessed comorbid illnesses, allowing us to estimate risk differences stratified by presence or absence of comorbid conditions. We found that patients treated for latent tuberculosis infection were more likely to have comorbid illnesses than the general population, particularly when elderly. This observation likely reflects recommendations in the Canadian Tuberculosis Standards to treat patients with latent tuberculosis infection only if there is an increased risk of developing active disease — often a comorbid illness. ${ }^{2}$ Also, the presence of comorbid illness increases the likelihood of health care use, which is a requisite for being screened and dispensed latent tuberculosis infection therapy.

\section{Conclusion}

Our study provides estimates of the risk of adverse events requiring hospital admission related to therapy for latent tuberculosis infection. These risk estimates are age-stratified, are based on virtually all patients treated among a large population during a period of six years, and account for comorbidities and the occurrence of similar health events in the general population. The risk of hospital admission due to adverse events is substantially increased in people over age 65 . These estimates could be useful for a re-analysis of the risks and benefits of therapy for latent tuberculosis infection in the elderly, which could influence recommendations for therapy in this group. In the absence of such an analysis, our data suggest that the risks of therapy for latent tuberculosis infection are considerable among the elderly and should be considered very carefully before therapy is given.

\section{References}

1. Targeted tuberculin testing and treatment of latent tuberculosis infection. Joint statement of the American Thoracic Society, the Centers for Disease Control and the Council of the Infectious Diseases Society of America. Am J Respir Crit Care Med 2000;161(suppl4, pt 2):S221-47.

2. Long R, Ellis E, editors. Canadian tuberculosis standards. 6th ed. Ottawa (ON): The Canadian Lung Association, Canadian Thoracic Society, Public Health Agency of Canada and Tuberculosis Prevention and Control; 2007.

3. Schwartzman K. Latent tuberculosis: old problem, new priorities. CMAJ 2002;166:759-61.

4. Centers for Disease Control and Prevention. Reported tuberculosis in the United States, 2007. Atlanta (GA): US Dept of Health \& Human Services; 2008

5. Sorresso DJ, Mehta JB, Harvill LM, et al. Underutilization of isoniazid chemoprophylaxis in tuberculosis contacts 50 years of age and older. A prospective analysis. Chest 1995;108:706-11.

6. Ferebee SH. Controlled chemoprophylaxis trials in tuberculosis: a general review. Bibl Tuberc 1969;17:28-106.

7. Kopanoff DE, Snider DE, Caras GJ. Isoniazid-related hepatitis: a US Public Health Service cooperative surveillance study. Am Rev Respir Dis 1978;117:991-1001.

8. Saukkonen JJ, Cohn DL, Jasmer RM, et al. An official statement: hepatotoxicity of antituberculous therapy. Am J Respir Crit Care Med 2006; 174:935-52.

9. Comstock GW. Evaluating isoniazid preventive therapy: the need for more data. Ann Intern Med 1981;94:817-9.

10. Riska N. Hepatitis cases in isoniazid treated groups and in a control group. Bull Int Union Tuberc 1976;51:203-8.

11. Salpeter SR. Fatal isoniazid-induced hepatitis: its risk during chemoprophylaxis. West J Med 1993;159:560-4.

12. Fountain FF, Tolley E, Chrisman CR, et al. Isoniazid hepatotoxicity associated with treatment of latent tuberculosis infection: a 7 -year evaluation from a public health tuberculosis clinic. Chest 2005; 128:116-23.

13. Nolan CM, Goldberg SV, Buskin SE. Hepatotoxicity associated with isoniazid preventive therapy: a 7-year survey from a public health TB clinic. JAMA 1999;281:1014-8.

14. LoBue PA, Moser KS. Use of isoniazid for latent tuberculosis infection in a public health clinic. Am J Respir Crit Care Med 2003;168:443-7

15. Moulding T. Toxicity associated with isoniazid preventive therapy. JAMA 1999;282:2207-8

16. Population and dwelling counts, for Canada, provinces, territories and census subdivisions (municipalities), 2006 census. Ottawa (ON). Statistics Canada; 2007.

17. Tamblyn R, Lavoie G, Petrella L, et al. The use of prescription claims databases in pharmacoepidemiological research: the accuracy and comprehensiveness of the prescription claims database in Quebec. J Clin Epidemiol 1995;48:999-1009.

18. Tamblyn R, Reid T, Mayo N, et al. Using medical services claims to assess injuries in the elderly: sensitivity of diagnostic and procedure codes for injury ascertainment. J Clin Epidemiol 2000;53:183-94

19. Update: Fatal and severe liver injuries associated with rifampin and pyrazinamide for latent tuberculosis infection, and revisions in the American Thoracic Society/CDC recommendations United States 2001. JAMA 2001;286:1445-6.

20. Deyo RA, Cherkin DC, Ciol MA. Adapting a clinical comorbidity index for use with ICD-9-CM administrative databases. $J$ Clin Epidemiol 1992;45:613-9.

21. Garibaldi RA, Drusin RE, Ferebee SH, et al. Isoniazid-associated hepatitis - report of an outbreak. Am Rev Respir Dis 1972;106:357-64.

22. Black M, Mitchell JR, Zimmerman HJ, et al. Isoniazid-associated hepatitis in 114 patients. Gastroenterology 1975;69:289-302.

23. Snider DE Jr, Caras GJ. Isoniazid-associated hepatitis: a review of available information. Am Rev Respir Dis 1992;145:494-7.

24. Millard PS, Wilcosky TC, Reade-Christopher SJ, et al. Isoniazid-related fatal hepatitis. West J Med 1996;164:486-91.

Affiliations: From the Respiratory Division (Smith, Schwartzman, Menzies) and the Respiratory Epidemiology and Clinical Research Unit (Schwartzman, Menzies), Montreal Chest Institute, McGill University; and the Department of Family Medicine (Bartlett), McGill University Health Centre, McGill University, Montréal, Que.

Contributors: Dick Menzies, Kevin Schwartzman and Gillian Bartlett were involved in the conception and design of the study. Gillian Bartlett was involved in the acquisition of the data, and Benjamin Smith, Dick Menzies and Kevin Schwartzman were involved in the analysis and interpretation of the data. Benjamin Smith was involved in the drafting of the manuscript, and Benjamin Smith and Gillian Bartlett were involved in the revision of the manuscript. All of the authors had full access to the data, and all of them approved the final version of the manuscript submitted for publication.

Funding: Financial support for this study was provided by an operating grant from the Canadian Institutes of Health Research (CIHR) (grant no. MOP-74710) and grants from the Fonds de la recherche en santé du Québec (a Chercheur boursier career award to Gillian Bartlett, a Chercheur boursier-clinicien career award to Kevin Schwartzman and a Chercheur National career award to Dick Menzies). These funding bodies were not involved in the conduct of the study. 